\title{
Development of Manufacturing Control and Data Acquisition System for Micro Electrochemical Milling Process
}

\author{
Yong $\mathrm{Liu}^{1,2}$, Jianhua Zhang ${ }^{2, *}$ and Huitao Cai ${ }^{1}$ \\ ${ }^{I}$ Associated Engineering Research Center of Mechanics \& Mechatronic Equipment, Shandong University, Weihai, \\ 264209, China \\ ${ }^{2}$ School of Mechanical Engineering, Shandong University, Jinan, 250061, China
}

\begin{abstract}
In order to realize real-time control and detection of the three-dimensional micro-electrochemical milling process, a data acquisition and control system is established based on virtual instrument software LabWindows/CVI. The three-dimensional tool path optimization, tool path servo feed algorithm, the data acquisition and anti-jamming algorithm are investigated. First, according to the characteristics of micro-electrochemical milling process, this paper analyzes the requirements of the control and detection system in depth. After that, a high-precision experimental system for 3D microelectrochemical milling is established. Then, the optimization of tool path and the data acquisition and its feedback control module are discussed as follows. Finally, experiments are carried out and a three-step staircase structure is fabricated successfully. It shows that the system can meet the requirements of micro-electrochemical milling process.
\end{abstract}

Keywords: Data acquisition, Feedback control, LabWindows/CVI, Micro electrochemical milling, Milling by layer.

\section{INTRODUCTION}

In recent years, with the development of micro-electromechanical system (MEMS), demands for micro metal parts and products are growing rapidly in many fields, such as electronics, optics, medical, automotive, biotechnology, communications and aviation industry. The current micro fabrication methods mainly include LIGA/LIGA-Like technology, micro laser machining technology, micro-EDM technology and so on. These methods constitute the group of technologies for metal microstructure processing. Scholars from various countries using the above techniques have made many significant achievements [1-6].

In recent years, many researchers paid special attention to electrochemical micromachining (EMM) technology, and expected to solve the problem of micro metal parts fabrication by using its unique processing principle and characteristics. Many studies have attempted electrochemical micromachining. German scientists Rolf Schuster et al. fabricated a micro cavity with several ten micrometers [7], United States scientists Trimmer et al. processed a complex patterns with the structural size of sub-microns [8], South Korea's Kim et al. worked out three-dimensional micro structures with good surface roughness $[9,10]$. Kurita in Japan using electrochemical micromachining technology processed out of the micro-prismatic electrode a complex micro structure [11], B. Bhattacharyya in India machined the micro holes and the ushaped slot structures successfully [12].

The key techniques of electrochemical micromachining process, which mainly affects the machining accuracy

\footnotetext{
*Address correspondence to this author at the 17923 Jingshi Road, Jinan. China. Postcard: 250061; Tel: 086-18854168635; E-mail: rzliuyong@163.com
}

include: the fabrication of microelectrodes technology, the feeding device for electrochemical micromachining, the ultra-short pulse power technology and the real-time monitoring technology. In order to realize three-dimensional micro electrochemical milling with high precision, the electrochemical micromachining system must have real-time control and data acquisition module, which should have the advantages of real-time controlling, quick-response to abrupt change of machining current, good stability, anti-interference ability and so on.

In this paper, a data acquisition and control system for micro electrochemical milling is established with precise motion axis, servo motor, current sensors, motion control card, data acquisition card and other key equipments based on virtual instrument software LabWindows/CVI. The threedimensional tool path generation and control strategy, the data acquisition and anti-jamming algorithm, the machining time error compensation algorithm of the system are also investigated. Experiments show that the system can meet the requirements of micro electrochemical milling, such as high machining precision, fast response, accuracy control, reliability and stability, etc.

\section{PRINCIPLE OF EMM AND SYSTEM CONFIGU- RATION}

\subsection{Principle of EMM}

Based on the principle of electrochemical anodic dissolution, electrochemical micro-milling used the micro cylindrical electrode, by controlling its tool path, then finished the fabrication of the workpiece in electrolyte $[9,13,14]$. For complex structures or three-dimensional high aspect ratio cavities machining, a milling by layer process can be selected. The machining principle is shown in Fig. (1). 


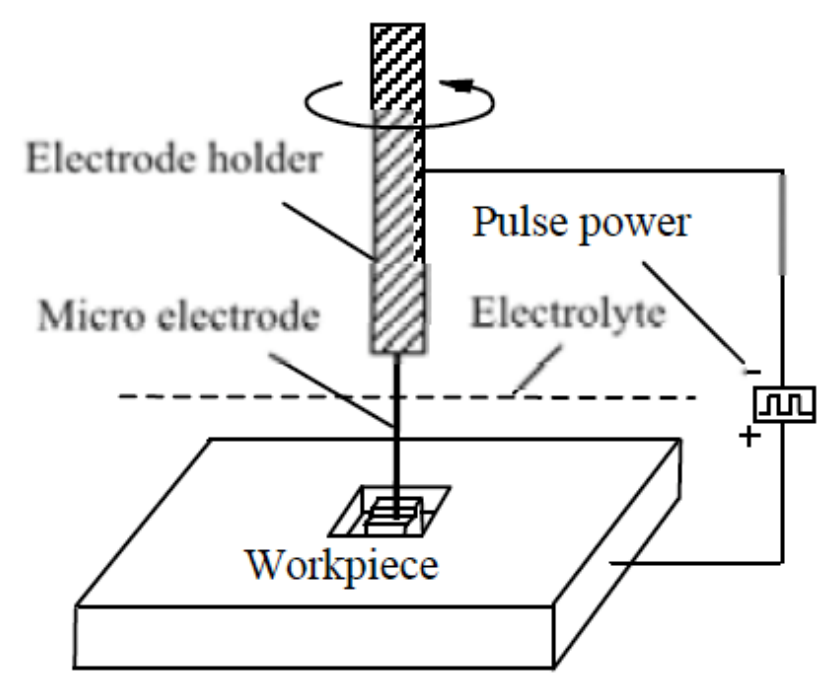

Fig. (1). Principle of micro electrochemical milling.

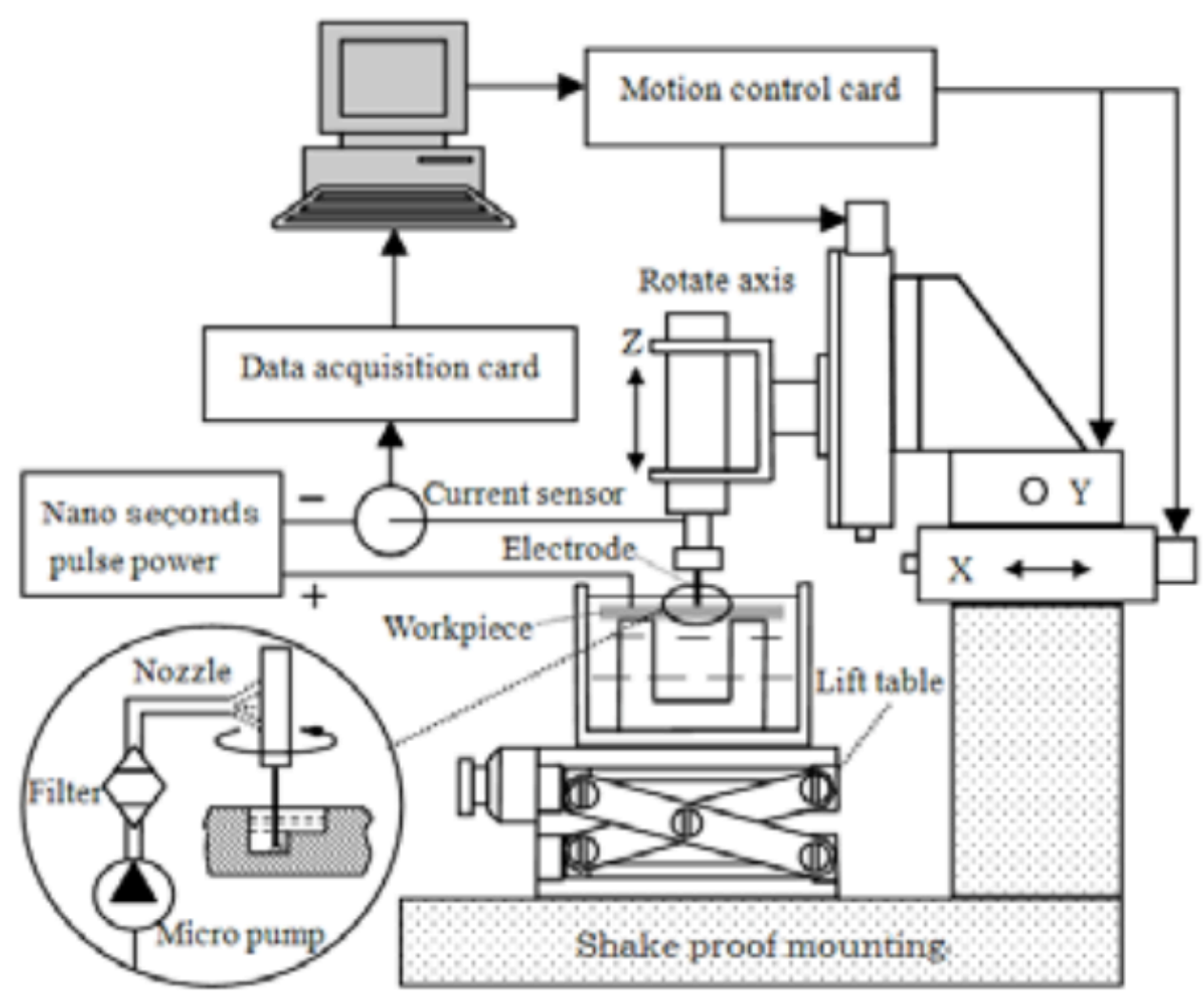

Fig. (2). Schematic of the entire EMM setup.

Electrochemical Micro-Milling platform demands high isolation and rigidity, so small vibration will seriously affect the processing process. For an electrode, which is just a few microns in diameter, the micro motion module must keep a good quality low-speed movement in the machining process, and cannot afford a crawling phenomenon or short-circuit. In order to ensure the machining accuracy and stability, the machining gap should be controlled in the feed resolution of 0.5 microns or less. Due to the fact that the machining gap is very small and cannot be measured directly, so the process requires real-time detection and processing signals to reflect the gap state. When a short circuit occurs, the servo motor will stop immediately and move back along the tool path quickly until the danger signal is clear.

\subsection{Machining Control and Detection System Hardware Configuration}

This paper established a three-dimensional electrochemical micro-milling experiment platform. The proposed EMM system, as shown in Fig (2), mainly includes nanosecond pulse power unit, the electrode system, the motion control 


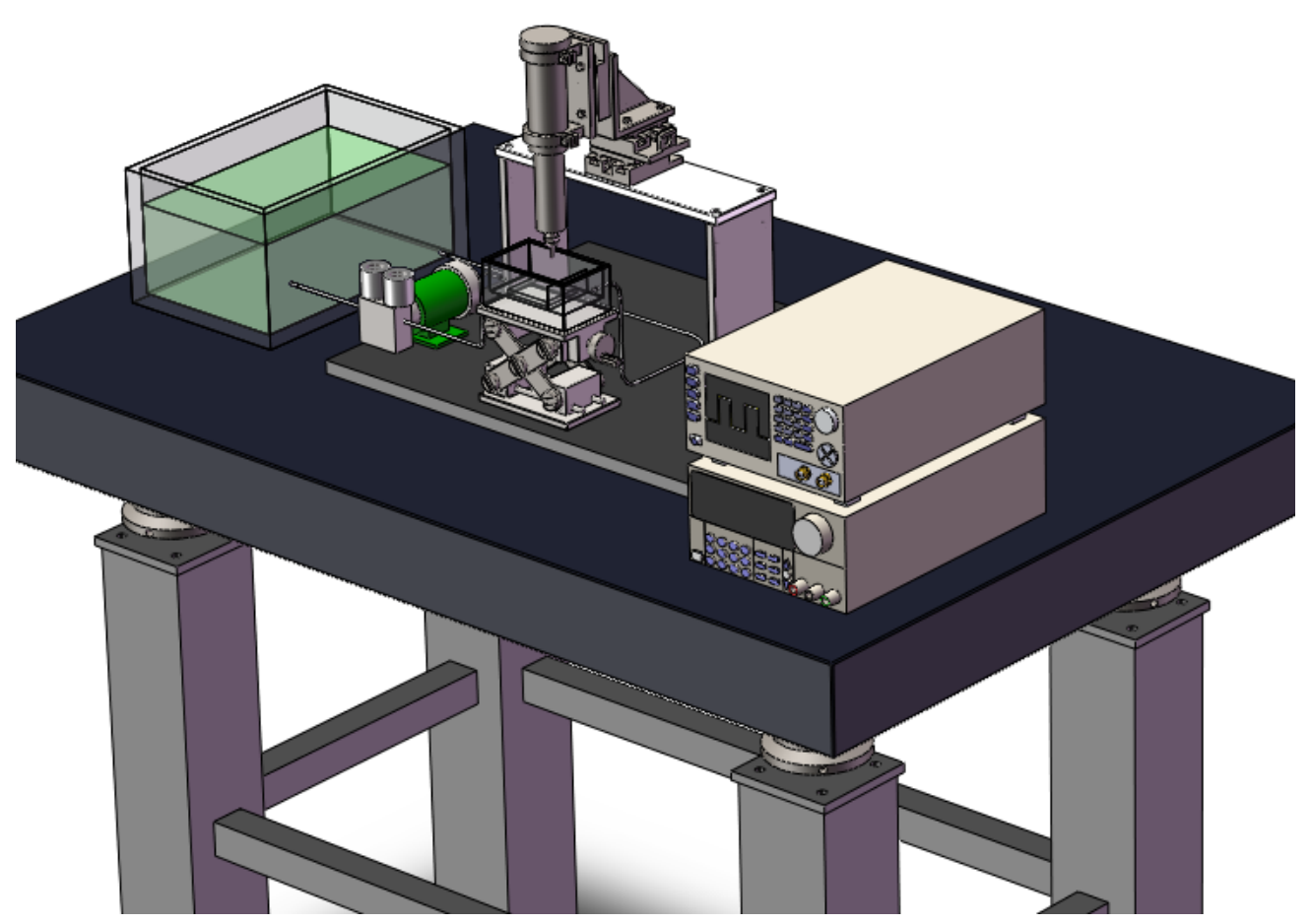

Fig. (3). Entity model of machine tool.

system, the machining state detection system, and the electrolyte circulating system. The Entity model of machine tool is shown in Fig. (3).

Electrode system consists of tool cathode, electrode clamps, high-speed spindle and the workpiece. Micron grade cylindrical tungsten electrodes are selected as tool cathode, which is connected by a screw thread between tool holder and the spindle. The high-speed spindle is clamped on the $\mathrm{Z}$ axis, which can move along with the $\mathrm{X}, \mathrm{Y}$, and $\mathrm{Z}$ axis. Workpiece is fixed in the electrolyte tank, which is mounted on the lifting platform and can be moved up and down. In the experiments, the positive and negative poles of the nanosecond pulse generator are connected to the workpiece and tool electrode, respectively. Low concentration of acid solution was selected to flow in circulatory system.

Motion control system consists of X, Y and Z, the three linear motion axis and the rotation axis $\mathrm{C}$, which are driven by motor drives. Rotation axis $\mathrm{C}$ is controlled by VFD-B programmable controller, whose speed can be continuously adjusted from 0 to 40000 RPM and with a radial run-out within 1 micron. Linear motion platform, a Adlink company (Taiwan) production, with MP-C154 four axis motion control card as the core, can control the GMT (Taiwan) electric sliding tables CYS-6020 (X, Y direction) and CXS-6030 (Z direction), and produce the three directions precision reciprocating feed of $0.25 \mu \mathrm{m} / \mathrm{step}$ resolution and reciprocating positioning accuracy of $\pm 0.5 \mu \mathrm{m}$, to meet the requirements of electrochemical micromachining.

The nanosecond pulse generator is selected as the power supply in the experiments, with a peak voltage output of $\pm 10 \mathrm{~V}$, and the minimum pulse width of $8 \mathrm{~ns}$. Detection system is mainly responsible for the current signal change which directly reflects the machining state. A small ohmic value resistor is connected in series in the circuit, and then the sensor detects the sampling resistance voltage value, which can be converted to the corresponding current value. Meanwhile, the multifunction data acquisition card NI PCI6221 input the detected signals to the computer through the $\mathrm{A} / \mathrm{D}$ converter. By the analysis of the data processing in computer, the real-time detection and feedback of machining status occurs. Composition of the process control and detection system is shown in Fig. (4).

\subsection{Software System Design and Workflow}

Three-dimensional microstructures machining can be programmed automatically in the CAM software, such as MasterCAM, UG, etc. to generate tool paths, which can be imported in the CNC system of EMM, and perform the complex three-dimensional structural milling by layer. The overall scheme of machining system, as shown in Fig (5), realizes the CAD/CAM integration of the EMM. The machining control and detection software system interface is shown in Fig. (6).

The automatic programming module is the core of the whole system, which mainly solves problems of the graphics data acquisition, automatic tracing, automatic processing direction discrimination, automatic tool path interpolation, and so on. Based on LabWindows/CVI virtual instrument environment, a machining control system software using a modular structure is developed, which consists the machining of a control module, data acquisition module and human-computer interaction and so on. The system can read 


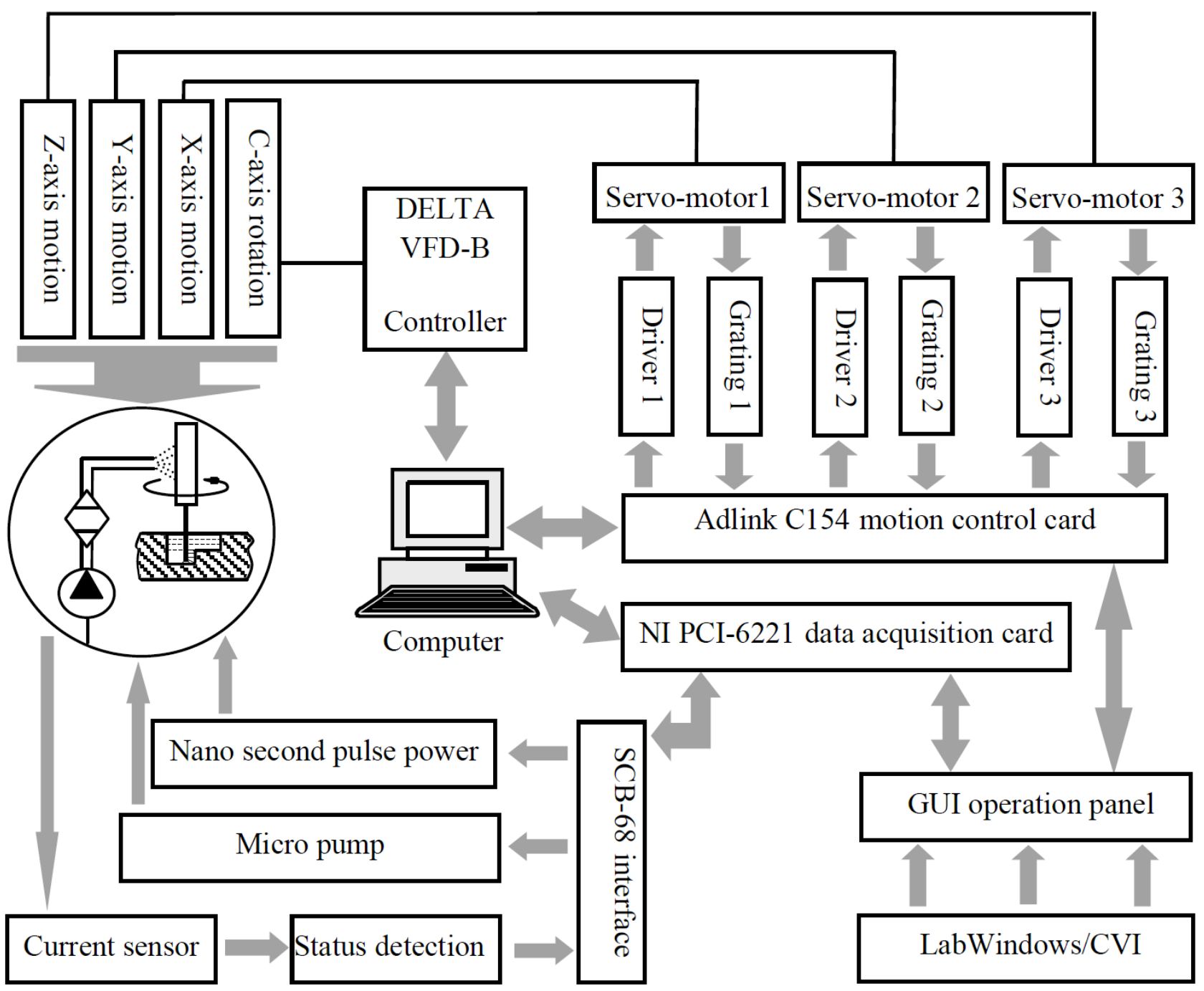

Fig. (4). Sketch of control and detection system.

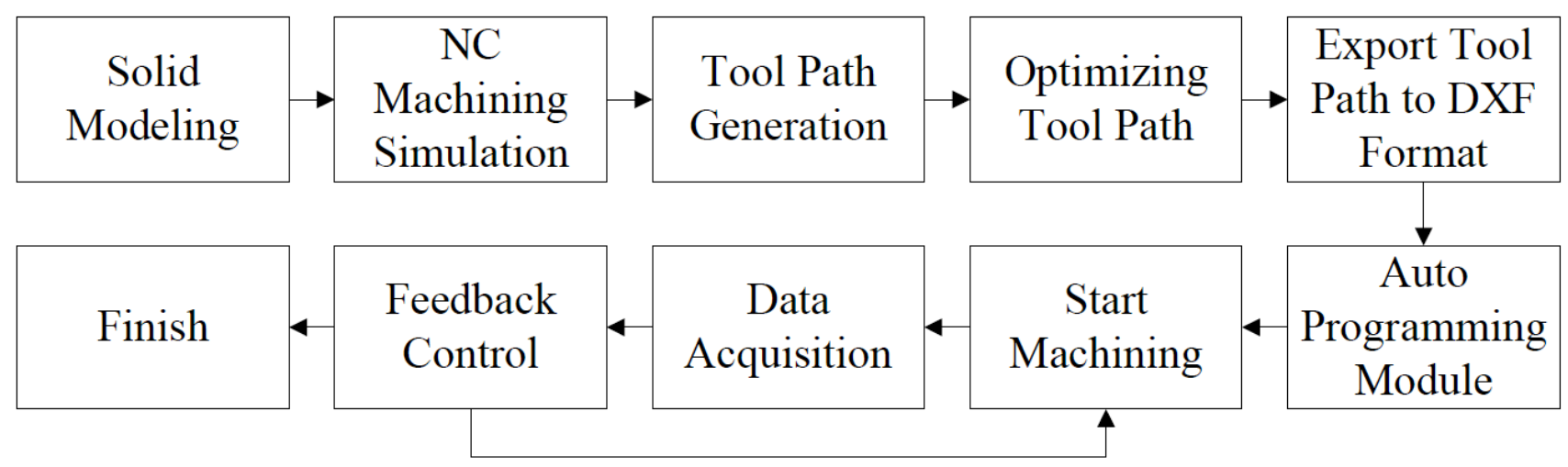

Fig. (5). Overall program of machining system. 


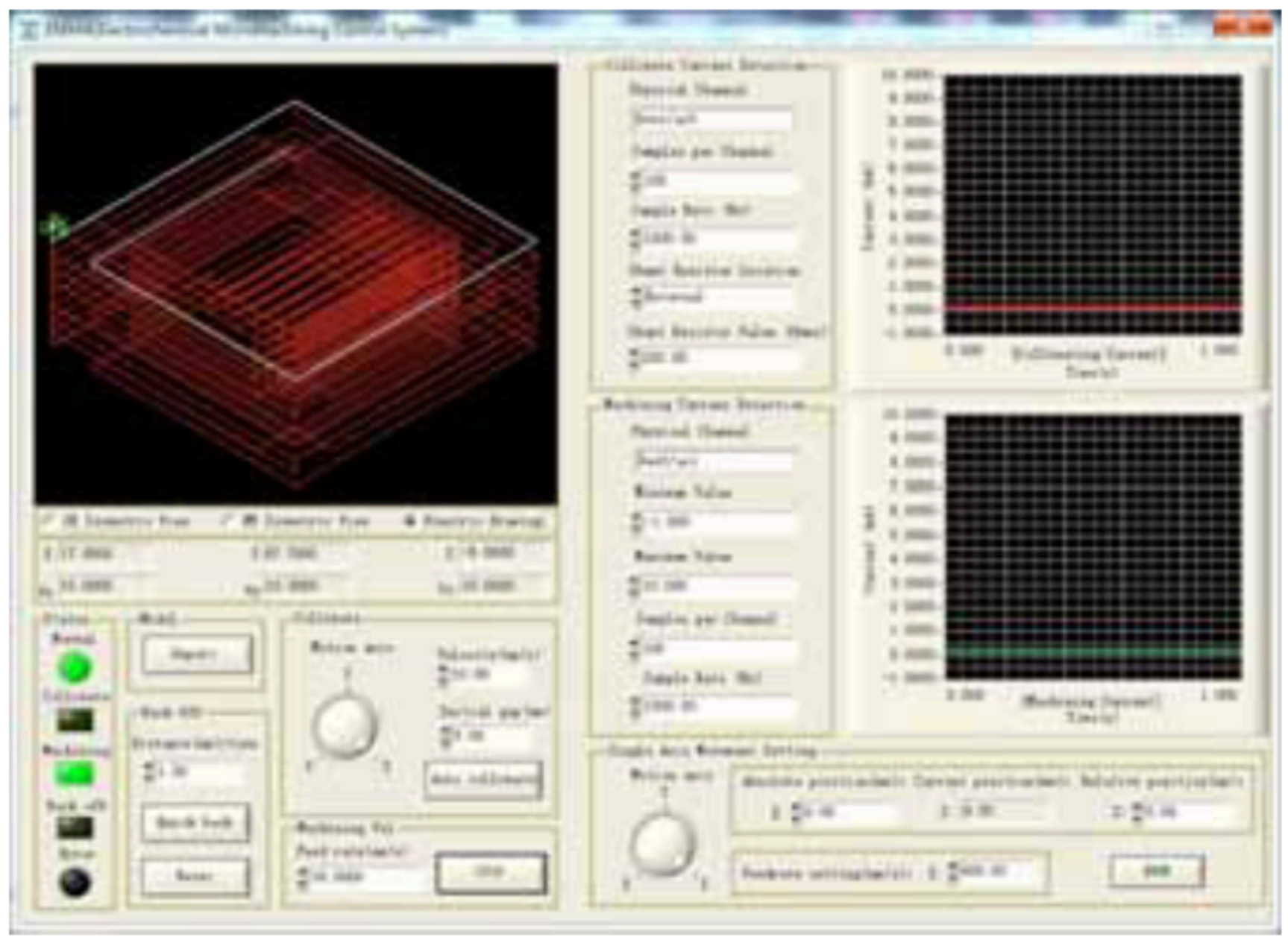

Fig. (6). Control and detection system software interface

three-dimensional solid model data from DXF drawing file directly, and then control servo feeding of the electrode in accordance with the tool path in real time.

\section{KEY STRATEGIES AND ALGORITHMS OF SOFTWARE SYSTEM}

\subsection{Tool Path Optimization}

In general, tool path generated by the CAM software needs to be edited and modified. Especially for complex parts, the unnecessary tool lift-off in automatic tool path may appear dozens or even hundreds of times for the curved surface machining. In addition, the cut mode or feed direction in the automatic tool path may not be reasonable or practical for machining, so it needs to be changed.

For electrochemical micromachining, the result of tool path optimization directly affects the machining precision. Each start point of automatic tool path in hierarchical milling process often overlaps which will be machined by the electrode sidewall by electrolysis and will seriously influence the shape precision of the point. In our tool paths optimization, each hierarchical point should spread out to avoid duplicated feeding over the same position. Fig. (7a) shows a 3D model to be machined, and the automatic tool path by the CAM software is shown in Fig. (7b), from which we can see, multiple tool lift-off occurs in the automatic tool path, and the start point for each layer overlaps. To ensure the shape precision of EMM, the automatic tool path should be optimized. The optimized result is shown in Fig. (7c), which removes the unnecessary lift-off, spreads out each hierarchical point and changes the cut mode and direction of feed in some places.

\subsection{Tool Path Servo Feed Algorithm}

The system can directly identify DXF graphic data file and servo feed by point to point control. DXF File consists of four Sections and end of file (EOF) flag, the sequencing of which is HEADER, TABLES, BLOCKS, ENTITIES, and the EOF flag. In the above five sections, the information related to the geometry is contained in the entity section. So other sections can be ignored in the process of reading the entity information.

Entity section consists of several groups, each group includes two rows. The first row is GROUP CODE, the second row is the GROUP VALUE. Group code is used to describe data types and the Group value is used to describe entity specific numerical values. Take the straight line for example, A group of codes $(10,20,30)$ represents the coordinates of starting point $(\mathrm{X} 1, \mathrm{Y} 1, \mathrm{Z1})$, a group of code $(11,21,31)$ il- 


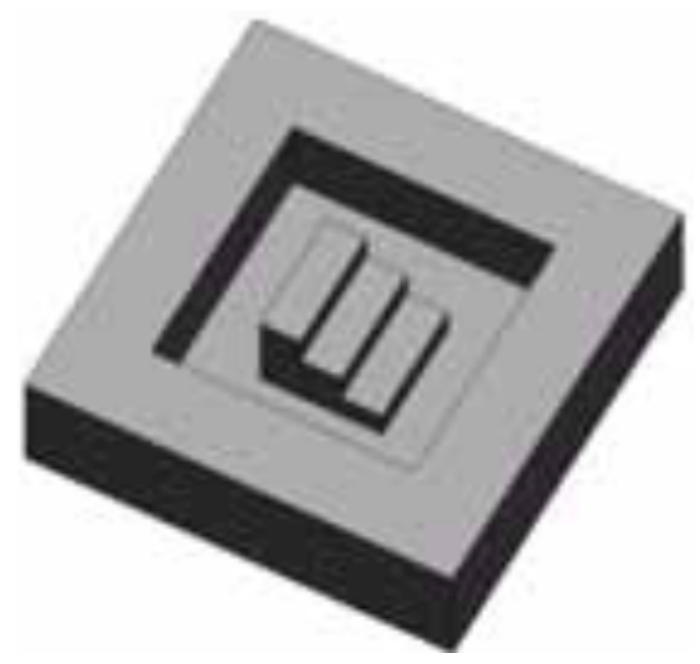

(a) Solid model

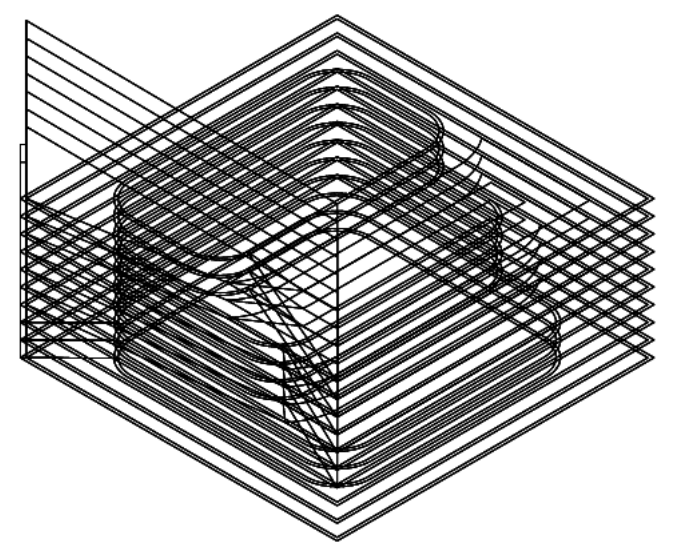

(b) Default Tool path

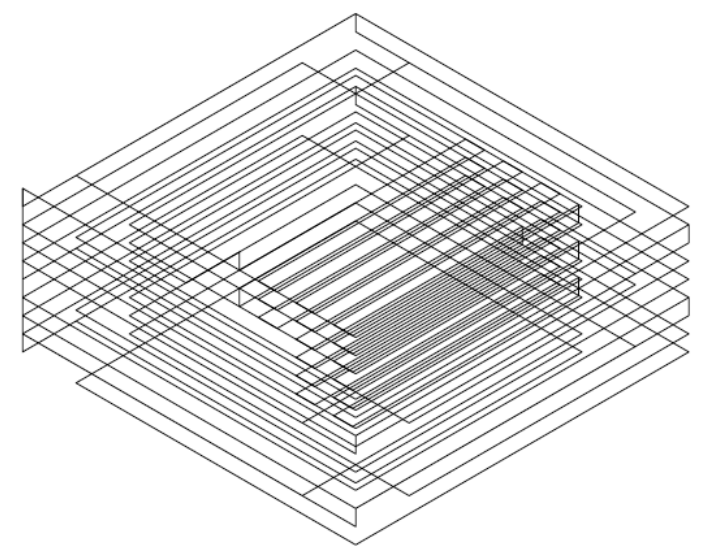

(c) Optimized tool path

Fig. (7). Solid model and its tool path.

lustrates the coordinates of end point (X2, Y2, Z2). For the arc, a group of codes $(10,20,30)$ represents the coordinates of circle center (X0, Y0, Z0), code 40 is the arc of the radius, and the codes $(50,51)$ represent the arc starting angle and the end angle, respectively. Based on above, we designed a program interface for reading DXF data file by using $\mathrm{C}$ programming language, the program flow is shown in Fig. (8).
After reading out the entity information of DXF file, which is sorted and generated in the entity chained list. And then interpolation operation with high precision is applied for each entity section to generate a series of machining position point end to end. In EMM process, the movement direction of electrode is controlled by the machining chained list. When short-circuit occurs, the electrode needs to move back 


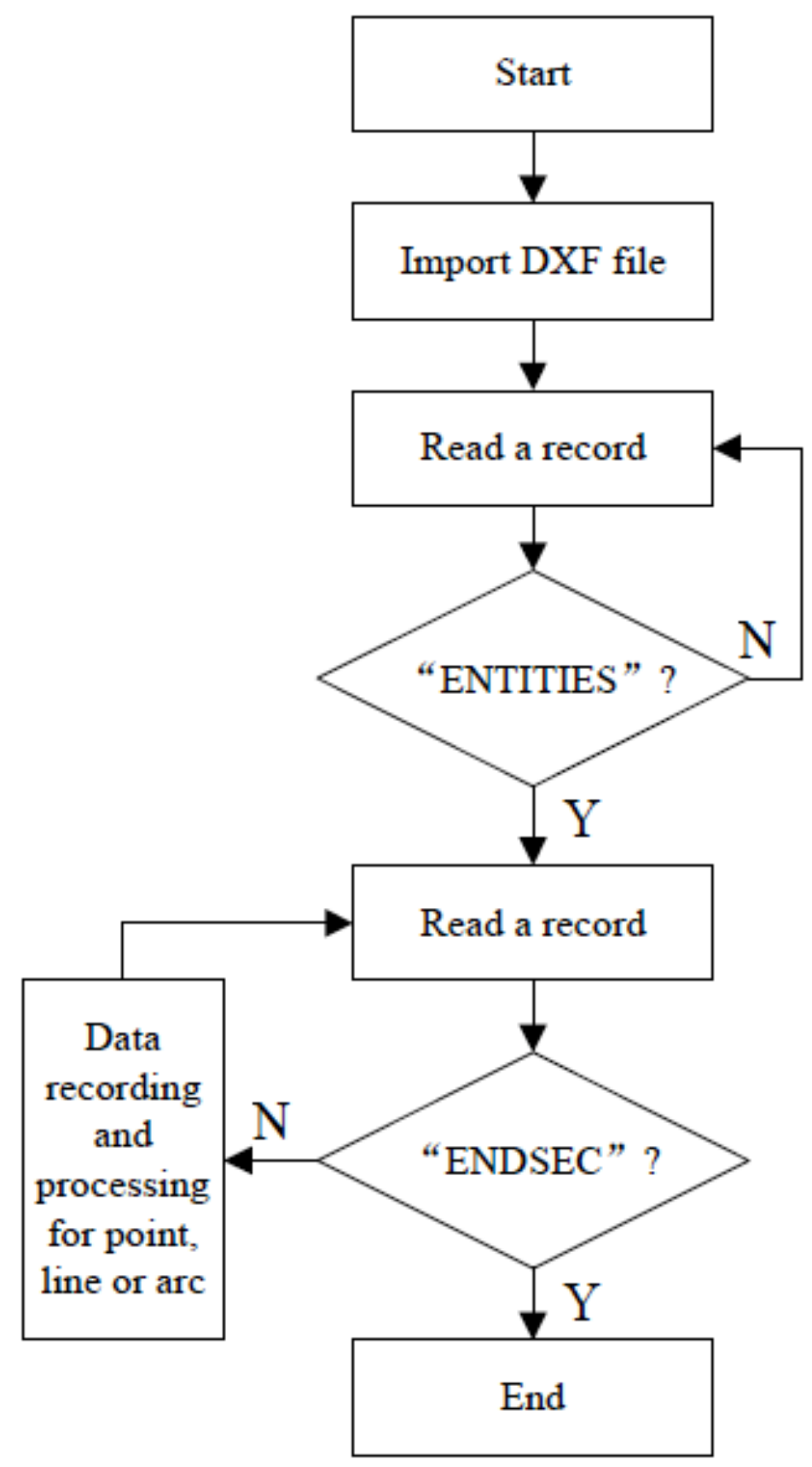

Fig. (8). Flow of reading entity information.

quickly by the original feed tool path. So the control system must have positive and reverse bidirectional interpolation function. Fig. (9) is bidirectional feeding flow chart for tool path control. The machining position chained list is designed as bidirectional chained list data structure, and each node includes the node data, a pointer to the previous node, prev, and the pointer to next node, next, so as to realize the bidirectional interpolation of tool path. Machining position chained list is the data-structure to store all the coordinates of the points in the tool path, which controls the electrode movement accurately in order to obtain the required shape and dimension, and it is the core of the whole of motion control for the tool path.

\subsection{Data Processing and Feedback Control Algorithm}

Both the machining gap state of EMM and the machining stability have great influence on the machining quality. In electrochemical micromachining with nanosecond pulse process, short-circuit phenomenon would occur if the machining gap is too small. So the status of the machining gap needs real-time detection. But the machining gap with micron scale is too small to be measured directly. In actual machining process, the detection system is used to detect changes in the circuit signal and current values, which indirectly reflects the machining state. If an abnormal signal is detected, the servo motor will stop immediately and move back along the tool path quickly until the danger signal is clear. 


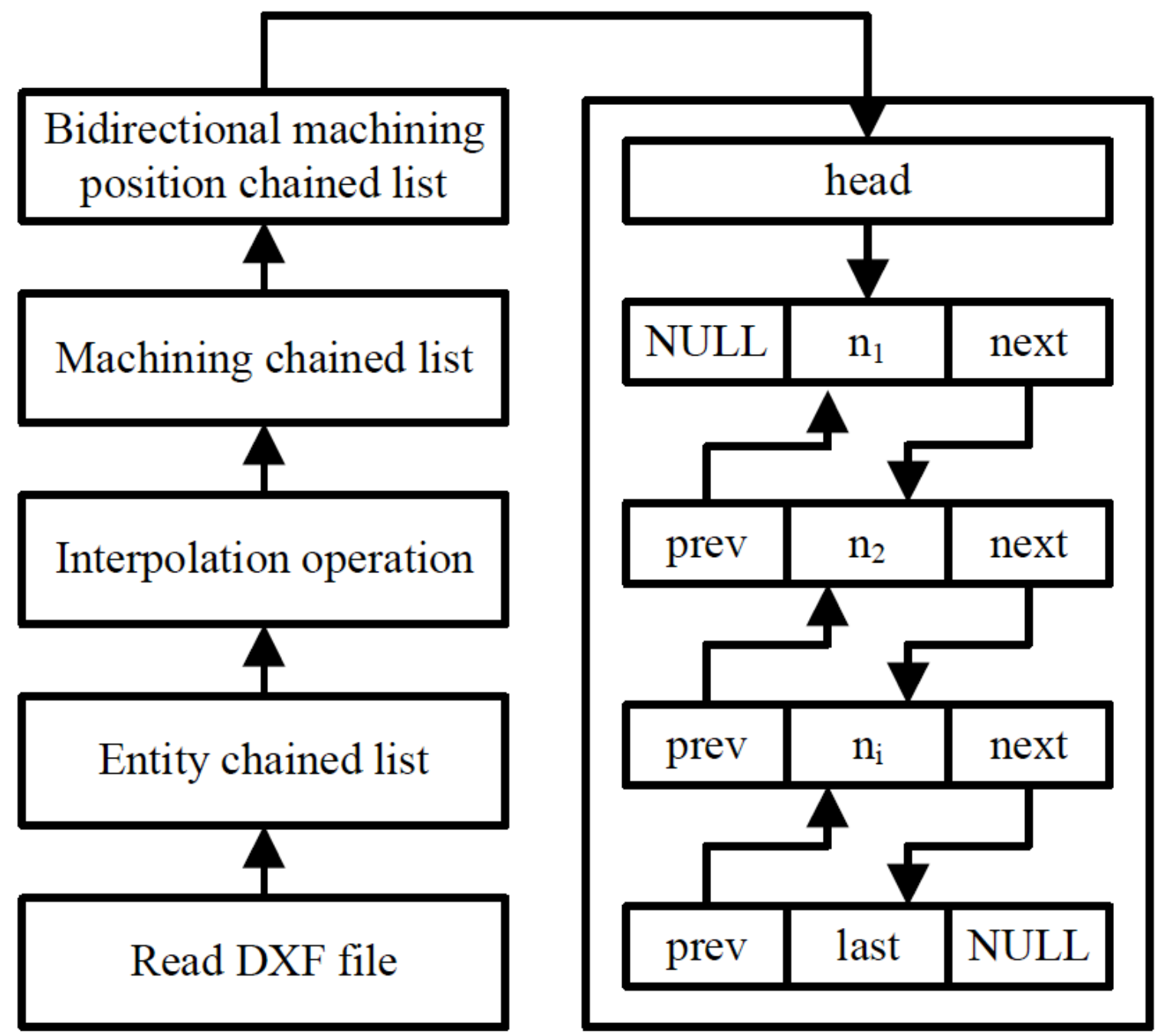

Fig. (9). Flow of tool path bidirectional feed control.

In this paper, the real-time current signal is measured to reflect the status of the machining gap. The data acquisition card PCI 6221 (National Instrument company) is selected as the core of the detection system, the A/D conversion time of which is only $6 \mu \mathrm{s}$, and the sampling frequency is $333 \mathrm{KS} / \mathrm{s}$ with strong real-time performance. Based on LabWindows/CVI virtual instrument environment, the DAQmx library functions can be called easily to complete the data acquisition task.

In EMM process, the real-time current signal is detected to reflect the changes of the machining gap and to prevent the short circuit phenomenon. The theoretical calculation value of the current in the balance gap is considered as the current threshold.

$I=\frac{\kappa(U-\delta E) \cdot A}{\Delta}$

$\Delta_{b}=\frac{\eta \omega \kappa(U-\delta E)}{v}$

Where $\mathrm{k}$ is the electrolyte conductivity, $\mathrm{U}-\delta \mathrm{E}$ is the voltage between electrodes, $\mathrm{v}$ is the feed rate, $\eta$ is the current efficiency, $\omega$ is the volume electrochemical equivalent, I is the machining current, $\mathrm{A}$ is the machining area.

Based on the above two equations, the machining gap $\Delta b$ can be calculated as follows:

$I_{b}=\frac{v A}{\eta \omega}$

Where, the calculated current $\mathrm{I}_{\mathrm{b}}$ is considered as a threshold current, which is compared with the machining current I to judge the machining status. If, $I \geq I_{b}$, then the servo motor will stop immediately, and move back along the tool path quickly until, $\mathrm{I}<\mathrm{I}_{\mathrm{b}}$.

The current value of electrochemical micromachining with nanosecond pulse is generally below $1 \mathrm{~mA}$, which is very small and is susceptible to interference from the external noise. In practical machining, the current data is interfered by the electromagnetic noise from the spindle rotation, and appears as a noise waveform, as shown in Fig. (10). Due to the existence of the noise current, the detection system may generate an error to the feedback control. After analysis, the electromagnetic noise has a certain regularity cycle, in 


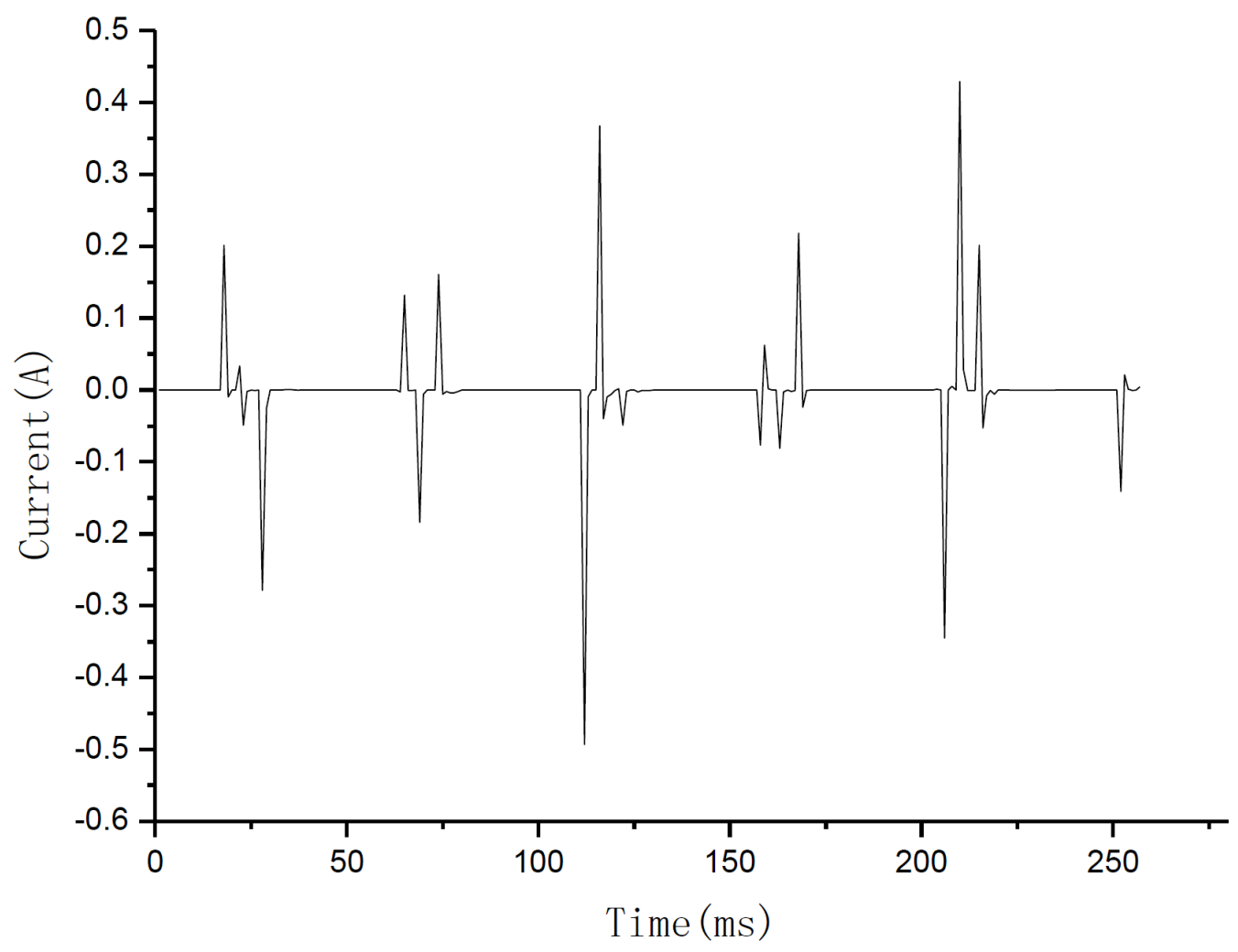

Fig. (10). Current waveform with noise.

which the duration of each period of the noise waveform is not greater than $15 \mathrm{~ms}$. Based on this rule, we designed the anti-jamming algorithm to distinguish the mutation current caused by the noise or by the short circuit. The default sampling frequency is set to $1000 \mathrm{~Hz}$, and the sampling point per unit time is set to 1000 .

Based on the principle of median filter, the current signal is operated by a period of $15 \mathrm{~ms}$. The expression is as follows:

$\bar{I}=\frac{1}{15} \sum_{i=1}^{15}\left|I_{i}\right|$

Where, $I$ is the mean current value for a period, $I_{i}$ is current in each sampling data point.

After the above treatment, the noise signal duration will not be more than two continuous periods. So, the noise and short circuit signals can be distinguished by the following judgment algorithm:

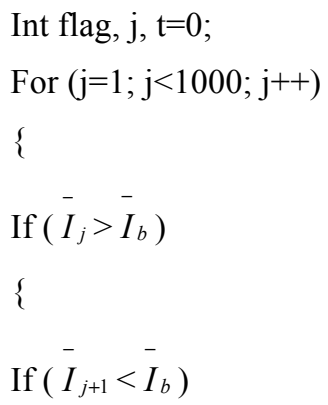

$$
\left\{P_{t}=\bar{I}_{j+1} ; \mathrm{t}=\mathrm{t}+1 ; \text { flag }=0 ;\right\} / / \text { Shield } \bar{I}_{j} \text { and Flag normal }
$$

Else if $\left(\bar{I}_{j+1}>\bar{I}_{b}\right) \& \&\left(\bar{I}_{j+2}<\bar{I}_{b}\right)$

$\left\{P_{t}=\bar{I}_{j+2} ; \mathrm{t}=\mathrm{t}+1 ;\right.$ flag $\left.=0 ;\right\} / /$ Shield $\bar{I}_{j}, \bar{I}_{j+1}$, Flag normal

else $\{$ flag=1;break; $\} / /$ Short circuit and Flag abnormal

\}

else $\left\{P_{t}=\bar{I}_{j} ; \mathrm{t}=\mathrm{t}+1 ;\right\}$

\}

If abnormal data appears continuously consecutively, the signal is generally considered as a noise, which will be shielded, otherwise the signal is considered as a short circuit. Meanwhile, the servo motor will stop immediately and move back along the tool path quickly until the danger signal is cleared.

\section{EXPERIMENTS AND RESULTS}

In this paper, micro cylindrical tungsten electrode with diameter of $10 \mu \mathrm{m}$ was prepared as a cathode tool to machine the object structure as shown in Fig. (7a). The experiment of electrochemical micro-milling by layer was carried out on 


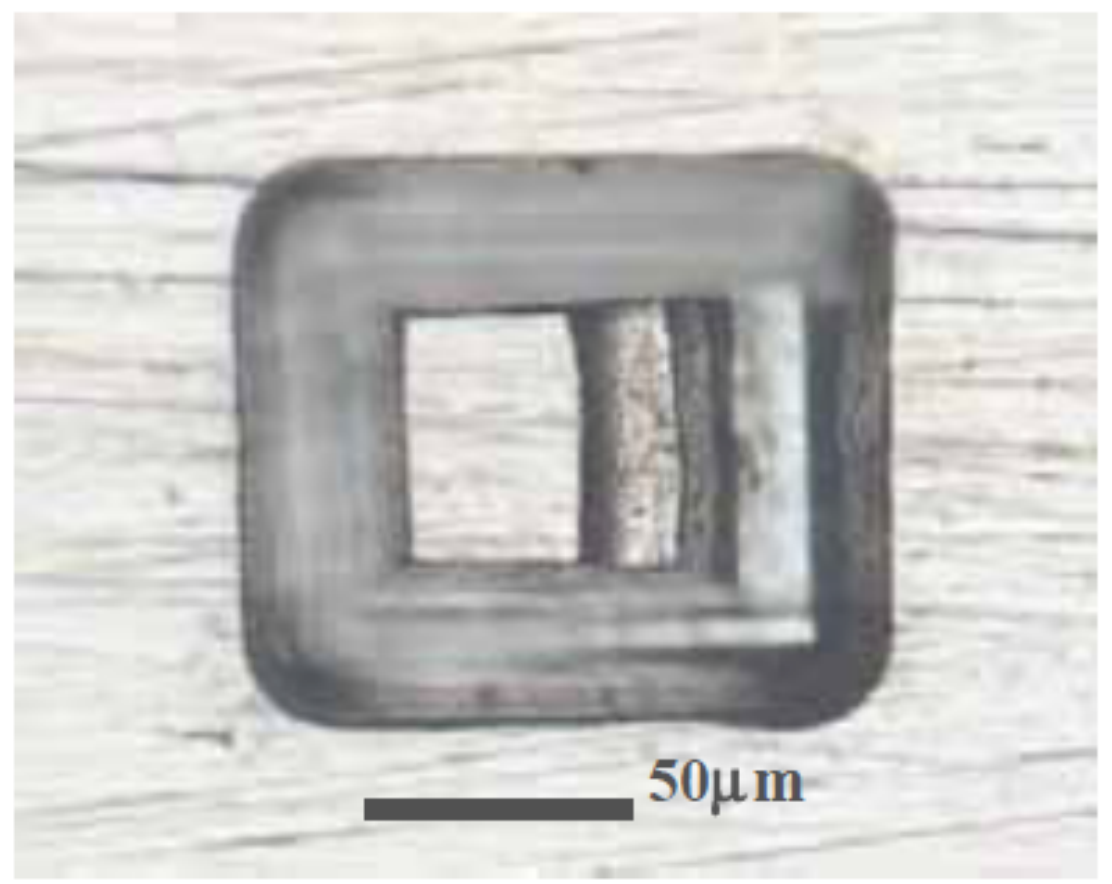

Fig. (11). Micro structure with three steps.

the $300 \mu \mathrm{m}$ thick nickel based superalloy (GH3030). The machining parameters in details are as follows: $0.2 \mathrm{Mol} / \mathrm{L}$ H2SO4 electrolyte, machining voltage of $4.5 \mathrm{~V}$, pulse width of $95 \mathrm{~ns}$ and pulse period of $1 \mu \mathrm{s}$. The experimental result is shown in Fig. (11), which has good shape accuracy with total depth of about $45 \mu \mathrm{m}$.

\section{CONCLUSION}

In this paper, according to the requirements of electrochemical micro-milling technology with high precision and fast response, a data acquisition and control system for EMM is established based on virtual instrument software LabWindows/CVI. And then, the three-dimensional tool path optimization, tool path servo feed algorithm and the data acquisition and anti-jamming algorithm are investigated. Finally, a 3D structure with three steps was machined successfully, which has greater aspect ratio and good shape precision. It shows that the manufacturing system can meet all the requirements of EMM adequately, such as high precision, fast response, stability in operation, and reliability in performance.

\section{CONFLICT OF INTEREST}

The authors confirm that this article content has no conflict of interest.

\section{ACKNOWLEDGEMENTS}

Authors acknowledge financial support from the China Nature Science Foundation (No. 51305238) and the Independent Innovation Foundation of Shandong University, IIFSDU (2012ZRYQ009).

\section{REFERENCES}

[1] K. Takahata, and Y. B. Gianchandani, "Batch mode microelectro-discharge machining", Microelectromech. Syst., vol. 11, pp. 102-110, 2002.

[2] W. S. Zhao, Y. Z. Li, Z. L. Wang, Y. Yang, and Y. Zhang. "Research on Key Techniques of 3D Micro-EDM Milling", Microfabricat. Technol., vol. 9, no. 3. pp. 49-55, 2003.

[3] P. M. Ming, D. Zhu, Y. Y. Hu, and Y. B. Zeng, "Fabrication of nickel soft contact microprobe based on UV-LIGA", Optics Precision Eng., vol. 15, no. 5, pp. 735-740, 2007.

[4] W. H. Huang, L. F. Zhu, Y. H. Cheng, M. Zhou, F. Yan, and X. Wang, "AFM based femto-second laser nanofabrication of PMMA”, Optics Precision Eng., vol. 15, no.12, pp. 1959-1962, 2007.

[5] X. H. Zheng, and D. Zhu, "Micro electroforming of metal microstructure array", Precision Eng., vol. 16, no. 3, pp. 473-477, 2008.

[6] Y. Zhao, Y. C. Liang, Q. S Bai, B. Wang, Y. Sun, and M. J. Chen, "Micro-milling machine tool, micro-tool wear and cutting forces in micro-machining", Precision Eng., vol. 15, no. 6, pp. 894-902, 2007.

[7] R. Schuster, V. Kirchner, P. Allongue, and G. Ertl, "Electrochemical micromachining", Science, vol. 289, pp. 98-101, 2000.

[8] A.L. Trimmer, Electrochemical Machining of Micro and Nanostructures with Ultra-Shot Voltage Pulse. University of Virginia: Virginia, 2005.

[9] A.H. Kim, S.H. Ryu, D.K. Choi, and C.N. Chu, "Micro electrochemical milling", J. Micromech. Microeng., vol. 15, no. 1, pp. 24-129, 2005.

[10] A.H. Kim, C.W. Na, Y.S. Lee, D.K. Choi, and C.N Chu, "Micro electrochemical machining of 3D micro structure using dilute sulfuric acid", Ann. CIRP, vol. 54, no. 1, pp. 191-194, 2005.

[11] T. Kurita, and K. Chikamori, "A study of three-dimensional shape machining with An EC $\mu \mathrm{M}$ system", Int. J. Mach. Tools Manuf., vol. 46, no. 12, pp. 1311-1318, 2005.

[12] B. Ghoshal, and B. Bhattacharyya, "Micro electrochemical sinking and milling method for generation of micro features," Proceedings of the Institution of Mechanical Engineers, Part B: J. Eng. Manuf., vol. 227, no. 11. pp. 1651-1663, 2013. 
[13] X. H. Li, Z. L. Wang, and W. S. Zhao, "The electrochemical micromachining based on multifunction tool for micromachining", J. Shang Hai Jiao Tong Univ., vol. 40, no. 6, pp. 909-913, 2005 .
[14] Z. Y. Zhang, D. Zhu, and M. H. Wang, "Theoretical and experimental research into electrochemical micro-machining using nanosecond pulses", Chin. J. Mech. Eng., vol. 43, no. 1, pp. 208$213,2007$.

Received: September 16, 2014

Revised: December 23, 2014

Accepted: December 31, 2014

(C) Liu et al.; Licensee Bentham Open.

This is an open access article licensed under the terms of the Creative Commons Attribution Non-Commercial License (http:/ creativecommons.org/licenses/by-nc/4.0/) which permits unrestricted, non-commercial use, distribution and reproduction in any medium, provided the work is properly cited. 